\title{
TUBERCULOSE PULMONAR: LEVANTAMENTO EPIDEMIOLÓGICO NO CONJUNTO PENITENCIÁRIO DE JEQUIÉ - BA
}

\section{LUNG TUBERCULOSIS: EPIDEMIOLOGICAL SURVEY IN A PENITENCIARY UNIT IN JEQUIÉ - BA}

\author{
Murilo Oliveira Ferreira de Britto ${ }^{1}$ \\ Maria Graziélle Bossi da Silva² \\ Bárbara Santos Ribeiro ${ }^{3}$ \\ Carine de Jesus Soares ${ }^{4}$ \\ Rafael da Silva Passos ${ }^{5}$
}

O presente trabalho objetivou estudar dados relacionados à prevalência da tuberculose, a partir de levantamento feito no Conjunto Penitenciário de Jequié-BA. Os dados foram analisados com base no Livro de Registro da referida unidade prisional, considerando o diagnóstico de positividades para a doença, pelo método de baciloscopia. Os resultados abrangem o período de julho de 2009 a janeiro de 2015 e apresentam a prevalência de infecção e de casos de tuberculose, caracterizando, na população exposta, o risco de infecção ou reinfecção. Entre janeiro de 2009 a janeiro de 2015, o percentual de amostras positivas foi de $6,2 \%$ das amostras analisadas. No ano de 2012, observou-se a maior concentração de amostras com alta contaminação de bacilos por campo, além de um aumento vertiginoso na prevalência/positivação de casos no Conjunto Penitenciário, quando comparados aos resultados obtidos nas Unidades de Saúde do Município, aumento este de $5.590,0 \%$, observados nos anos de 2012 e de 2014. Entretanto, notou-se que a maioria dos pacientes com baciloscopia positiva foram tratados, resultando na cura. Infere-se que a precariedade nas condições de vida dos detentos pode ter contribuído para a alta prevalência dessa doença, ratificada pela diferença nos percentuais de positividade de casos, quando comparados os valores obtidos nos exames da população encarceirada com os demais indivíduos do município que fizeram os exames. Assim, torna-se necessária a busca de outras formas de abordagem para um programa de tuberculose "adequado", além dos dados encontrados servirem de alerta para a sociedade, para que as possíveis medidas sejam tomadas.

Palavras-chave: Bacilo de Koch. Baciloscopia. Conjunto Penitenciário. Mycobacterium tuberculosis. Tuberculose Pulmonar.

The present study aimed to study data related to the prevalence of tuberculosis, based on a survey carried out in the Jequié-BA Penitentiary Unit. Data from the Registry Book of the prison unit were analyzed, considering the diagnosis of positivitiy for the disease by the bacilloscopy method. The results cover the period from June 2009 to January 2015 and present the prevalence of infection and cases of tuberculosis, characterizing the risk of infection or reinfection of the exposed population. Between the years of 2009 and January 2015 the percentage of positive samples was 6.2\% of the samples analyzed. In the year 2012 and 2014 the highest concentration of samples with high contamination of bacilli per field was observed, as well as a vertiginous increase in the prevalence / positivation of cases in the penitentiary complex, when compared to the results obtained in the health units of the municipality, representing an increase of 5.590.0\%. However, it was noticed that the majority of patients with positive smear microscopy were treated, resulting in cure. The precariousness of the prisoners' living conditions may have contributed to the high prevalence of this disease, confirmed by the difference in the percentages of positive cases in the incarcerated population when compared to the other individuals in the municipality who did the exams. Thus, it is necessary to search for other forms of approach to

\footnotetext{
${ }^{1}$ Faculdades Unidas de Pesquisa, Ciências e Saúde (FAPEC); Jequié; Bahia; Lattes: http://lattes.cnpq.br/0790287440812332 murilo83biomed@hotmail.com

${ }^{2}$ Faculdades Unidas de Pesquisa, Ciências e Saúde (FAPEC); Jequié; Bahia; Lattes: http://lattes.cnpq.br/6181306706003144; grazibossi@hotmail.com

${ }^{3}$ Faculdades Unidas de Pesquisa, Ciências e Saúde (FAPEC); Jequié; Bahia; Lattes: http://lattes.cnpq.br/9652318818158869; barbara_ribeiro2@hotmail.com

${ }^{4}$ Faculdades Unidas de Pesquisa, Ciências e Saúde (FAPEC); Jequié; Bahia; Lattes: http://lattes.cnpq.br/3849567863954014; carineesoares@hotmail.com

${ }^{5}$ Faculdades Unidas de Pesquisa, Ciências e Saúde (FAPEC); Jequié; Bahia; Lattes: http://lattes.cnpq.br/2491340738506293; rafaelspassos08@gmail.com
} 
an "adequate"tuberculosis program.

Keywords: Koch Bacillus. Mycobacterium tuberculosis. Pulmonary Tuberculosis. Prision Set. Smear.

\section{INTRODUÇÃO}

A tuberculose é uma doença infecciosa causada pela bactéria Mycobacterium tuberculosis, que foi descrita em 1882, pelo bacteriologista alemão Robert Koch. Ele conseguiu isolar o microrganismo, que ficou mundialmente conhecido como bacilo de Koch em sua homenagem (BRASIL, 2010). Este bacilo tem sua forma de propagação pelo ar, através da fala, do espirro e, principalmente, da tosse, sendo sua transmissão direta, ou seja, de pessoa para pessoas, podendo aumentar ainda mais a probabilidade de contágio em ambientes fechados ou com má ventilação (OMS, 2013).

O risco de infecção depende da intensidade e da duração da exposição ao bacilo e de características como idade, estado imunológico e nutricional, doenças intercorrentes e condições socioeconômicas. Embora possa atingir quase todos os órgãos do corpo, a tuberculose afeta normalmente os pulmões, mas pode atingir o cérebro e os rins, provocando problemas como dificuldades respiratórias e derrame pleural (presença de líquido no espaço entre pulmão e tórax). O principal sintoma é a tosse frequente, mas também podem ser observados suor noturno e diminuição de apetite (MITCHELL et al., 2012).

Ao contrário da maioria das doenças infecciosas, a tuberculose possui manifestação tardia, levando em média de 4 a 12 semanas para que as primeiras lesões sejam descobertas. Estima-se que uma pessoa infectada possa contaminar de 10 a 15 pessoas por ano. A doença é tratada com uma combinação de antibióticos. Como a bactéria cresce lentamente, os medicamentos devem ser tomados por, pelo menos, 6 meses (PORTO, 2001). Dados recentes estimam que a tuberculose afetou 8,6 milhões de pessoas e causou 1,3 milhão de mortes no mundo, no ano de 2012 (OMS, 2013). A Organização Mundial de Saúde prevê que até 2 milhões de indivíduos apresentem a forma resistente da doença em 2015, representando um grande problema de saúde pública (BRASIL, 2010).

Como a tuberculose é uma patologia de fácil transmissão, as precárias condições de vida nas prisões, com padrões de higiene insatisfatórios, associados à aglomeração, com déficit nutricional, favorecem a ocorrência de adoecimento e morte. Além disso, algumas práticas contribuem ainda mais para a transmissão, como a intensa mobilidade da população carcerária entre uma prisão e outra, constituindo-se em um fator importante a ser observado, já que a interface no processo de expansão da doença favorece o ciclo transmissivo da $M$. tuberculosis, colocando em risco tanto a saúde dos presos quanto a dos demais constituintes da "população carcerária", que incluem familiares, agentes de segurança e profissionais de saúde (TODRYS et al., 2011).

A unidade prisional do município de Jequié custodia presos provisórios e condenados, dando cumprimento às penas privativas de liberdade, em regime fechado, semiaberto e aberto, com segurança máxima. Este presídio tem estrutura para comportar 416 detentos, porém, em 21 de maio de 2015 abrigava 1015 pessoas, entre presos de regimes semiaberto e fechado, além dos provisórios (GONÇALVES, 2016). Portanto, como a prevalência e a incidência da tuberculose pulmonar nas populações carcerárias são muito maiores do que na população em geral, a mensuração desses dados é de grande interesse para futuras intervenções.

Assim, é de extrema importância a difusão de informações sobre a patogenia, sobre a transmissão e sobre a situação da tuberculose nos presídios brasileiros, para que medidas de prevenção e de tratamento possam ser tomadas pelos órgãos responsáveis, a fim de que ocorra uma melhoria na qualidade de vida da população carcerária.

O presente trabalho teve por objetivo estudar dados relacionados à prevalência da tuberculose pulmonar, a partir de levantamento feito no Conjunto Penitenciário de Jequié-BA, relativos ao período de janeiro de 2009 a janeiro de 2015. 


\section{METODOLOGIA}

Constitui-se de um estudo descritivo transversal, através de um levantamento de dados epidemiológicos, descrevendo a magnitude dos problemas de saúde na população de estudo. Os dados relativos às avaliações, ações de prevenção, controle, tratamento e fatores etiológicos da tuberculose pulmonar foram retirados do Livro de Registro da população encarcerada na Unidade Prisional de Jequié-BA. A baciloscopia foi o método utilizado para determinação de positividade para a tuberculose na população exposta ao risco de infecção ou reinfecção.

O município de Jequié está localizado na região sudoeste da Bahia, distante cerca de $365 \mathrm{Km}$ da capital Salvador-BA, e abriga a referida unidade prisional (PMJ, 2017). Segundo a Secretaria da Administração Penitenciária (SEAP), em março de 2015, o sistema prisional do estado contava com 12.863 internos (SEP e SEAP, 2015). O presídio de Jequié-BA tem estrutura para abrigar 416 detentos, porém, mais de 1 mil pessoas (aproximadamente 150,0\% acima do ideal) se encontram encarceradas em regime semiaberto ou fechado, além dos provisórios, indicando superlotação (GONÇALVES, 2016).

O levantamento considerou os dados obtidos entre o período de janeiro de 2009 a janeiro de 2015 , levando em consideração a avaliação epidemiológica da população estudada, abordando quantitativamente os dados positivos e negativos observados nos exames de baciloscopia, conforme Livro de Registro da unidade, comparando-os com os dados disponíveis na literatura sobre a tuberculose pulmonar.

O Livro de Registro da unidade é parte de um material de padronização nacional, cedido pelo "Programa Nacional de Controle da Tuberculose", da Secretaria de Vigilância em Saúde, do Ministério da Saúde, e é intitulado "Registro de pacientes e acompanhamento de casos de tuberculose". Nele, constam os parâmetros considerados para os testes laboratoriais, tais como número do prontuário, número do Sistema de Notificação de Agravos e Notificação (SINAN), nome do paciente, idade e gênero, bem como os resultados dos exames de diagnóstico, como a baciloscopia de escarro, cultura, prova tuberculínica (PPD), histopatologia, radiografia $(R X)$ de tórax e outros exames (bioquímicos, ultrassonografia, $R X$ de outros órgãos, bacilo álcool-ácido resistente - BAAR - em outros órgãos e vírus da imunodeficiência humana-HIV).

Além destes parâmetros, constam ainda informações acerca das formas clínicas das referidas doenças, tipo de registro a ser lançado (casos novos, recidivas, retornos), tratamento, baciloscopia de acompanhamento, situações de encerramento (por motivos de cura, encerramento do tratamento, êxito no tratamento, abandono, transferência para outra unidade, óbito, falência ou mudança de diagnóstico) e número de contatos (registrados e examinados).

Apesar dos dados supracitados, foram utilizados apenas os dados elencados em uma página específica do livro de registros, que apresenta a "Avaliação dos resultados e tratamento". Nesta parte, estão os dados referentes ao "Estudo de coorte", a saber: forma de tratamento, unidade de saúde, município, unidade federativa (UF), período analisado e uma tabela com os dados, nas linhas: casos novos, retorno após abandono, recidiva e falência; e nas colunas: forma clínica, casos avaliados, cura, completou o tratamento, êxito no tratamento, abandono, transferência, óbito e falência.

Como a pesquisa foi realizada apenas sobre os dados dos "resultados e tratamento", que estavam devidamente preenchidos pelo servidor responsável, não ocorrendo contato algum dos pesquisadores com os detentos, podemos afirmar que nesta pesquisa não houve risco iminente para os pesquisadores. $\mathrm{E}$, como apenas foram utilizados os dados dessa tabela na presente pesquisa, e esta não contém os nomes dos pacientes/encarcerados, tornou-se dispensável a aplicação do Termo de Consentimento Livre e Esclarecido (TCLE), uma vez que seus nomes não foram expostos. No entanto, este projeto foi submetido aprovado pelo Comitê de Ética em Pesquisa da Universidade Estadual do Sudoeste da Bahia (CEP-UESB), sob CAAE 34622114.2.0000.0055 e parecer 884.291.

O diagnóstico da tuberculose pulmonar é baseado em duas baciloscopias diretas positivas no exame de escarro e uma cultura positiva para $M$. tuberculosis. Esta baciloscopia é uma técnica padronizada que consiste do esfregaço de uma amostra clínica (de secreção biológica - escarro), fixada em lâmina que é submetida à técnica de Ziehl-Neelsen baseada na capacidade das bactérias de 
incorporar e reter determinados corantes que identificam as bactérias ácido-álcool-resistentes (BAAR). Trata- se de um método diagnóstico importante devido a sua sensibilidade, configurada através da positividade de diagnósticos, chegando a $80 \%$ de casos bacilíferos (pacientes com baciloscopia positiva) - ou seja, este teste possui uma sensibilidade de cerca de $80 \%$, apresentando ainda um custo baixo e execução simples (BRASIL, 2010, BRASIL, 2005).

Aanálise estatística foi realizada com o auxílio do software Epi Info (3.3.2) e Microsoft Excel 2010.

\section{RESULTADOS EDISCUSSÃO}

A baciloscopia de escarro em presidiários do Conjunto Penitenciário de Jequié-BA, registrados no Livro de Registro, permitiu observar que, especificamente nos anos de 2010 e de 2015, não houve registro de positividade nas amostras analisadas. Entre os anos de 2009 e janeiro de 2015, o percentual de amostras positivas foi de $6,2 \%$ do total de amostras analisadas, ou seja, 33 amostras positivas, de um total de 497 exames.

Segundo a administração, a unidade prisional pesquisada está ocupada por mais de 1.000 detentos, sendo que em torno de $10 \%$ dessa população é constituída por mulheres.

Em relação ao número de exames de baciloscopia realizados no ano de 2009, observou-se uma diminuição; contudo, foi o ano de maior taxa de positividade, entre os estudados, com $25,0 \%$ de amostras positivas. Entretanto, no ano seguinte, 2010, não se observou positividade. Já em 2011, por sua vez, o percentual de positividade foi de 5,0\%. Em 2012, a média de exames realizados, quando comparada ao ano anterior, praticamente foi mantida, mas houve um aumento de $4 \%$ de casos positivos. No ano seguinte, 2013, houve uma redução de aproximadamente $10,0 \%$ na realização de exames nos pacientes/encarcerados, sem alteração da média de positividade. Em 2014, a quantidade de solicitações de exames de baciloscopia ultrapassou o dobro da observada no ano anterior, mas a média de positividade ainda se manteve como nos anos anteriores. Por fim, no ano de 2015 não foram observadas amostras positivas, sendo até o final da pesquisa realizadas 5 baciloscopias de escarro (Tabela 2).

Tabela 2. Registro de negatividade e positividade por ano para a baciloscopia de escarro em presidiários do Conjunto Penitenciários de Jequié, no período de julho de 2009 a janeiro de 2015.

\begin{tabular}{ccc} 
Baciloscopia de escarro & Negativos & Positivos \\
\hline 2009 & $75,0 \%$ & $25,0 \%$ \\
\hline 2010 & $100 \%$ & $0 \%$ \\
\hline 2011 & $95,0 \%$ & $5,0 \%$ \\
\hline 2012 & $91,0 \%$ & $9,0 \%$ \\
\hline 2013 & $93,0 \%$ & $7,0 \%$ \\
\hline 2014 & $95,0 \%$ & $5,0 \%$ \\
\hline 2015 & $100 \%$ & $0 \%$
\end{tabular}

Fonte: dados da pesquisa

Notou-se que, em 2014, a maioria dos pacientes com baciloscopia positiva foram tratados, o que levou à cura, como mostra a Tabela 3. 
Tabela 3. Tratamento dos bacilíferos do Conjunto Penal de Jequié. Ano de 2014.

\section{Acompanhamento das baciloscopias positivas}

\begin{tabular}{cc}
\hline Alta / Cura & $76,0 \%$ \\
\hline Alvará de soltura & $12,0 \%$ \\
\hline Abandono & $3,0 \%$ \\
\hline Transferências & $3,0 \%$ \\
\hline Óbito & $3,0 \%$ \\
\hline Suspensão & $3,0 \%$
\end{tabular}

Fonte: dados da pesquisa

No presente estudo, foi observado que os pacientes que mostraram positividade em suas amostras tinham entre 20 e 45 anos de idade, dados semelhantes aos obtidos por Oliveira e Cardoso (2004) no seu estudo, no qual a média de idade das amostras positivas foi de 28,7 , aproximando-se da relação jovem/adulto em privação de liberdade (BRASIL, 2012).

$\mathrm{Na}$ distribuição de casos novos por unidades federais, observa-se que os estados do Rio de Janeiro, Amazonas, Pernambuco, Pará, Rio Grande do Sul, Bahia, Ceará, Acre, Alagoas e Maranhão possuem incidências superiores a 38,2 casos por 100.000 habitantes. Alguns estados, como o Rio de Janeiro e o Amazonas, possuem elevadas incidências, com 71,7 e 66,9, respectivamente, sendo as maiores observadas (BRASIL, 2015).

Por outro lado, Goiás (8,6), Distrito Federal $(12,0)$ e Tocantins $(15,5)$ são estados que, historicamente, contam com os menores valores de incidência no país. A região Sudeste, principalmente o estado do Rio de Janeiro e de São Paulo, possui a maior quantidade absoluta de casos da doença no Brasil, enquanto que a região Norte possui a maior incidência quando comparada às demais regiões do país (BRASIL, 2015).

Na Bahia, no ano de 2006, ainda segundo dados do SINAN, a taxa de incidência de tuberculose (TB) alcançou 44,2 casos por grupos de 100 mil habitantes, sendo que o percentual de cura chegou a $67 \%$ e o abandono alcançou $6,1 \%$. O cenário epidemiológico da tuberculose no estado não apresentou mudanças significativas, pois a existência de bolsões de pobreza em todas as regiões do estado é fator determinante para a ocorrência da TB. Apesar disso, observa-se redução no número de casos e na taxa de incidência a cada ano, situação que também é notada nos dados do país. Entretanto, os estados de São Paulo, Rio de Janeiro e Bahia permanecem como os três estados com maior número de casos, representando $44,5 \%$ do total de casos novos no Brasil em 2013. Comparando com os demais estados do Nordeste, a Bahia contribuiu com $24,8 \%$ dos casos novos em 2013 , ocupando o $1^{\circ}$ lugar na região (BRASIL, 2014a).

No ano de 2012, observou-se a maior concentração de amostras com alta contaminação de bacilos por campo, 4 cruzes. No referido ano, dentre as amostras que foram positivas para a baciloscopia de escarro (9 amostras), 55,55\% (5 amostras) apresentaram mais de dez BAAR por campo, nos primeiros 5 campos microscópicos analisados, caracterizando o escalonamento em 4 cruzes (Gráfico 1).

A comparação entre os casos de positividade observados no conjunto penal e nas unidades de saúde do município está ilustrada na Tabela 4. As informações sobre a quantidade geral ano a ano foram colhidos na Secretaria Municipal de Saúde do município e cedidos pela diretora do Departamento de Vigilância Epidemiológica. Observou-se um aumento vertiginoso nos anos de 2012 e de 2014 na prevalência/positivação de casos no conjunto penitenciário, quando comparados aos resultados obtidos nas unidades de saúde do município, chegando esse aumento a expressivos $5.590,0 \%$. 
Gráfico 1. Concentração de BAAR (bacilo álcool ácido resistente) em amostras corada pelo método de ZiehlNeelsen do Conjunto Penitenciário de Jequié, entre julho de 2009 a janeiro de 2015.

\section{Gráfico Retrospectivos de Baciloscopias Positivas}

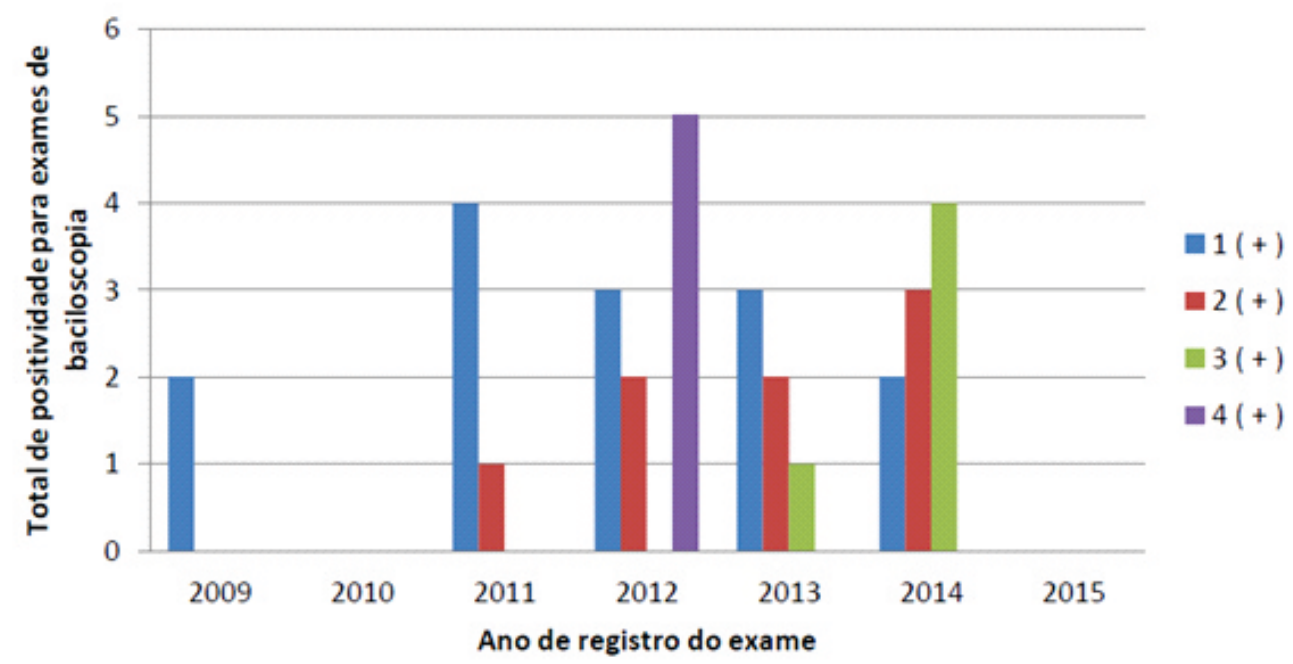

\footnotetext{
Nenhum BAAR em 100 campos observados Negativo

De um a nove BAAR por campo, em 100 campos observados Relata-se apenas a quantidade de BAAR

De dez a 99 BAAR por campo, em 100 campos observados ( + )

De um a dez BARR por campo, nos primeiros 50 campos observados $(++)$

Mais de dez BAAR por campo, nos primeiros 20 campos $(+++)$

Mais de dez BAAR por campo, nos primeiros 5 campos $(++++)$
}

Tabela 4. Tabela comparativa entre os exames de baciloscopia / BK no conjunto penitenciário e nas demais unidades de saúde do município de Jequié.

\begin{tabular}{cccc} 
Ano & $\begin{array}{c}\text { Conjunto Penal } \\
\text { (Dados } \\
\text { absolutos/casos por } \\
\mathbf{1 0 0} \text { mil habitantes) }\end{array}$ & Unidade de Saúde & $\begin{array}{c}\text { \% de casos no CP* } \\
\text { em relação às US }{ }^{* *}\end{array}$ \\
\hline $\mathbf{2 0 0 9}$ & $02 / 200$ & - & - \\
\hline $\mathbf{2 0 1 0}$ & - & 74 casos & - \\
\hline $\mathbf{2 0 1 1}$ & $05 / 500$ & 66 casos & $3.105 \% \uparrow$ \\
\hline $\mathbf{2 0 1 2}$ & $09 / 900$ & 35 casos & $5.590 \% \uparrow$ \\
\hline $\mathbf{2 0 1 3}$ & $05 / 500$ & 62 casos & $3.105 \% \uparrow$ \\
\hline $\mathbf{2 0 1 4}$ & $09 / 900$ & 71 casos & $5.590 \% \uparrow$ \\
\hline $\mathbf{2 0 1 5}$ & - & - & -
\end{tabular}

*CP: Conjunto Penal; **US: Unidade de Saúde

Fonte: dados da pesuisa

De acordo com a Organização Pan-Americana de Saúde, em 2008 a incidência de tuberculose nas unidades prisionais da América Latina foi 22,2 vezes maior que na população livre. Já no Brasil, a estimativa dessa incidência era 25 vezes maior, em 2011, que a prevalência de tuberculose ativa variando de $2,5 \%$ a 8,6\% entre os presos (BRASIL, 2011; OLIVEIRA; NATAL; CAMACHO, 2015). Porém, essa incidência pode estar subestimada, considerando a oferta e a qualidade do serviço de saúde no sistema prisional, a desmotivação dos profissionais e a percepção dos sintomas pelos presidiários, o que explicaria a baixa detecção de casos (OLIVEIRA; NATAL; CAMACHO, 2015; SÁNCHEZ, 2007).

O município de Jequié possui população estimada de 161.150 habitantes (PMJ, 2017), o que permite visualizar a exacerbação na quantidade de casos registrados no conjunto penal desse município, extrapolando, em dois anos (2012 e 2014), mais de 5 mil vezes a quantidade observada nas unidades de 
saúde.

A Portaria № 1.271, de 6 de junho de 2014, define a Lista Nacional de Notificação Compulsória de doenças, agravos e eventos de saúde pública nos serviços de saúde públicos e privados em todo o território nacional, e nela a tuberculose é tida como uma doença de notificação compulsória.

Pode-se observar neste trabalho que existem subnotificações e/ou inconsistências nos registros dos dados da doença, comprometendo seu diagnóstico e tratamento, além de contribuírem negativamente para a aquisição de maiores conhecimentos sobre a prevalência e a incidência desta doença na população, interferindo também na elaboração e na execução de programas de tratamento/controle e prevenção desta enfermidade, o que dificulta a eficácia dos programas de promoção à saúde.

De acordo com as recomendações da OMS, o controle da TB em prisões deve ser baseado, principalmente, como preconizado para a população livre, na Estratégia de Tratamento Supervisionado (DOTS), que engloba 5 componentes: compromisso político; identificação de casos bacilíferos, através de detecção passiva entre pacientes sintomáticos que buscamo serviço de saúde; quimioterapia de curta duração com acompanhamento do paciente, incluindo medicação supervisionada; suprimento regular de medicamentos; e sistema de registro e avaliação, incluindo resposta ao tratamento (OMS, 2013).

A OMS ainda recomenda que as ações para o controle da TB no sistema prisional contem com a integração entre os serviços penitenciários e extra penitenciários, com o acesso de todos os presos aos serviços de saúde e com suficiência de recursos estruturais, operativos, humanos, materiais e de transporte (OMS, 2013).

A superlotação na unidade pesquisada é um dos principais pontos para que se desenvolva uma epidemia. São várias as condições que contribuem para tal situação, como celas onde a circulação de ar é quase nula, falta de iluminação solar, crescentes taxas de ocupação e a alta prevalência de HIV. Outro problema é a quantidade insuficiente de profissionais de saúde que atuam nas prisões que, por serem mal remunerados e com contratos de trabalho precários, têm alta rotatividade (a mudança de funcionários da saúde é constante nestes setores).

O controle social dos serviços de saúde nas prisões aparenta estar malconduzido, a agregação de saúde e de justiça nos diferentes níveis de governo e sua articulação têm se mostrado insuficientes nas organizações da sociedade civil. Nesse sentido, são muitas as razões para a TB representar um urgente problema para as pessoas privadas de liberdade (DARA et al., 2009; BRASIL, 2011b; BRASIL, 2011c).

As pessoas vivendo com HIVIAIDS (PVHA) estão mais vulneráveis à tuberculose, sendo essa a principal causa definida de óbito entre as doenças infecciosas nesse grupo, o que justifica especial atenção às pessoas com coinfecção TB-HIV (OMS, 2013).

No Brasil, do total de casos novos diagnosticados em 2013, 59,2\% realizaram o teste para o diagnóstico de HIV. Nesse mesmo ano, o percentual de coinfecção TB-HIV no país foi de 9,8\%. Devido ao seu alto grau de complexidade, o desenvolvimento de programas de informação, educação e comunicação em prisões é limitado, em parte pelo quantitativo de detentos e pelo autoritarismo na segurança, que restringe a realização de atividades coletivas (BRASIL, 2014b).

\section{CONSIDERAÇÕES FINAIS}

A partir dos dados obtidos neste estudo, infere-se que a precariedade nas condições de vida dos detentos pode ter contribuído para a alta prevalência dessa doença, no presídio de Jequié-BA. Essa constatação é ratificada pela destacada diferença nos percentuais de positividade de casos, quando comparados os valores obtidos na população encarcerada e nos demais indivíduos que fizeram os exames nas unidades de saúde do município.

As improdutivas estratégias de educação prescritivas num meio altamente hierarquizado e que rejeita imposições torna necessária a busca de outras formas de abordagem para um programa de 
tuberculose "adequado", isto é, a utilização de regime terapêutico eficaz e a aplicação de medidas de vigilância, monitorando sua administração.

Os serviços de saúde penitenciários devem ter parceria com o Sistema de Saúde e a Justiça, reforçados nas esferas federal e estadual, atendendo às normas do contexto do Plano Nacional de Saúde no Sistema Penitenciário. Esse ajustamento de estratégias em função da realidade carcerária é fundamental para o controle da TB nas prisões brasileiras. Os dados encontrados neste estudo servem de alerta para a sociedade, para que medidas apropriadas sejam tomadas.

\section{REFERÊNCIAS}

BRASIL. Ministério da Saúde, Doenças Infecciosas e Parasitárias - Guia de Bolso. $8^{a}$ edição. Revista Brasília - DF, 2010.

Secretaria da Educação do Estado da Bahia (SEP); Secretaria de Administração

. Ministério da Saúde. Portaria n $^{\circ}$ 1.271, de 6 de junho de 2014. Disponível em:

<http://bvsms.saude.gov.br/bvs/saudelegis/gm/2014/prt1271_06_06_2014.html>. Acesso em: 15/07/2018.

Resolução $n^{\circ} 11$ do Conselho Nacional de Política Criminal e Penitenci ária, Ministério da Justiça do Brasil. Recomenda a Diretriz Básica para Detecção de Casos de Tuberculose entre ingressos no Sistema Penitenciário nas Unidades da Federação, Brasília/DF, 2011b.

Secretaria de Estado da Saúde. Coordenação dos Institutos de Pesquisa. Centro de Vigilância Epidemiológica Prof. Alexandre Vranjac. Divisão de Tuberculose e outras Pneumopatias. Recomendações para o controle da tuberculose nas prisões. São Paulo: Secretaria de Estado da Saúde. 199. Revista Nosso Século. São Paulo: Abril Cultural, n. 2, p. 1900-1910, 2011c.

Ministério da Saúde, Secretaria de Vigilância em Saúde, Boletim Epidemiológico. v. 45, n.2, 2014b.

. Manual de Recomendações para o Controle da Tuberculose no Brasil; Brasília/DF, 2011.

DARA, M. et al. Guidelines for control of tuberculosis in prisions, 2009 pdf.usaid.gov/pdf_docs/PNADP462.pdf. Acesso em: 25/03/2014.

GONÇALVES, Hilton de Miranda. A conversão religiosa como instrumento de tutela dos direitos fundamentais no conjunto penal de Jequié-Bahia. 2016. Disponível em:

<https://repositorio.ufba.br/ri/bitstream/ri/19725/1/Disserta\%C3\%A7\%C3\%A30\%20Final\%20-

\%20Hilton\%20de\%20Miranda\%20Gon\%C3\%A7alves.pdf>. Acesso em: 14/07/2018.

JEQUIÉ. Lei n 2.039, de 22 de dezembro de 2017. Ano II - ed. № 00448; caderno I. Disponível em:

<http://io.org.br/ba/jequie/arquivos_clientes/426/midia/152356.pdf>. Acesso em: 15/07/2018.

MITCHELL, R. n. et al. Fundamentos de Robbins \& Cotran: Patologia; tradução FERNANDES A. C. et al. Rio de Janeiro: Elsevier, 2012.

OLIVEIRA, L. G. D.; NATAL, S.; CAMACHO, L. A. B. Análise da implantação do Programa de Controle da Tuberculose em unidades prisionais no Brasil. Cadernos de Saúde Pública, v. 31, p. 543-554, 2015.

OLIVEIRA, Helenice Bosco de; CARDOSO, Janaina Corrêa. Tuberculose no sistema prisional de Campinas, São Paulo, Brasil. Revista Panamericana de Salud Pública, v. 15, p. 194-199, 2004. Disponível em:

<https://www.scielosp.org/scielo.php?pid=S1020-49892004000300008\&script=sci_arttext\&tlng=pt>. 
ORGANIZAÇÃO MUNDIAL DE SAÚDE - OMS. Global Tuberculosis Report, 2013. Disponível em: <HTTP://www.eho.int/tb/publications/global_report/es/>. Acesso em: 01/04/2014.

PORTO, C. C. Semiologia Médica. Rio de Janeiro. Editora Guanabara Koogan, 4ª Ed., 2001.

SÁNCHEZ, A. A. M. M. R., Tuberculose em População Carcerária do Estado do

Rio de Janeiro: prevalência e subsídios para formulação de estratégias de controle Rio de Janeiro, 2007.

TODRYS, K. W. et al.Preso e perigo: o acesso ao HIV e prevenção e tratamento e negação dos direitos humanos TB, nas prisões zamnianas. J Int AIDS SocN, 2011. 